\title{
Plan de cuidados estandarizado para pacientes de nefrología pediátrica
}

\author{
Pedro Bernal Herrera - Flora López González - María del Carmen Jerez Moreno - María del Carmen \\ Peinado Barraso
}

\section{Servicio de Nefrología Pediátrica. Hospital Infantil Virgen del Rocío. Sevilla}

\section{Sra. Directora:}

El Plan de Cuidados Estandarizado (PCE) es el instrumento que permite, facilita y optimiza la labor asistencial del enfermero, ofreciendo al paciente la calidad en el servicio y la unidad en la práctica clínica. En definitiva, el PCE es un protocolo específico de cuidados, apropiado para aquellos pacientes que padecen los problemas normales o previsibles relacionados con el diagnóstico concreto o una enfermedad. No debemos olvidar que los PCE servirán como modelo al enfermero y que necesariamente tendremos que personalizarlo para su aplicación concreta.

Entendemos como cuidados de enfermería en nefrología pediátrica, aquellos que tienen como objeto la atención integral del niño, la incorporación de la familia en su estudio y estrategia, promocionar el principio de autonomía y promover una atención individualizada y continua. Nuestra unidad de nefrología pediátrica atiende a niños desde el período neonato, en casos excepcionales, hasta los 16 años para:

- Estudio, diagnóstico y tratamiento

- Insuficiencia renal en período de prediálisis

- Diálisis peritoneal

- Hemodiálisis

- Trasplante renal

Durante este período el niño manifiesta signos y síntomas que pueden llegar a presentar patrones de salud alterados y en consecuencia producir una modificación en su demanda de cuidados.

\author{
Correspondencia: \\ Pedro Bernal Herrera \\ Plaza del Aljarafe, 6. Casa E \\ 41960 GINES (Sevilla) \\ E-mail:watapocco@telefonica.net
}

Los datos necesarios para la elaboración del PCE son recogidos mediante un registro de valoración inicial al ingreso del paciente (ya existente) que está basado en los patrones funcionales de Marjory Gordon y que configuran una serie de comportamientos, más 0 menos comunes a todas las personas, que contribuyen a su salud, calidad de vida y al logro de su potencial humano y que se dan de manera secuencial a lo largo del tiempo. Permiten una valoración enfermera sistemática y premeditada, con la que se obtiene una importante cantidad de datos del paciente de una manera ordenada lo que facilita el análisis de los mismos. Todo esto independientemente del modelo enfermero elegido, que en nuestro caso es el de Virginia Henderson que asocia un Diagnóstico Enfermero (DdE) a cada una de las necesidades de nuestros pacientes.

Partiendo de grupos de trabajo representados por componentes del Área de Pediatría, se elabora el PCE del paciente de nefrología pediátrica mediante evidencia científica y consenso. Son identificados los problemas más prevalentes en este tipo de pacientes utilizando la taxonomía NANDA (North American Nursing Diagnosis Association) de diagnósticos enfermeros para su descripción. Así mismo se establecen los resultados según taxonomía NOC (Nursing Outcomes Classification) que son valorados según la escalas de Likert (anexol), indicando la eficacia e idoneidad de las actividades propuestas para cada DdE. Se proponen intervenciones según taxonomía NIC (Nursing Interventions Classification). Por último se identifican los Problemas Interdisciplinares que potencialmente se puedan presentar en los pacientes de Nefrología Pediátrica.

Con este PCE potenciamos y agilizamos el uso de la taxonomía NANDA/NIC/NOC que nos permite disminuir la variabilidad clínica antes los mismos problemas de salud mediante una hoja registro que proponemos (anexo 2) en donde definimos 6 diagnósticos de enfermería, 7 resultados, 29 intervenciones y 5 complicaciones potenciales, que en la práctica supondrán 129 actividades potenciales. 
ANEXO 1. Escalas de Likert

\section{Escala Likert 1}

1. Nunca demostrado

2. Raramente demostrado

3. A veces demostrado

4. Frecuentemente demostrado

5. Siempre demostrado
Escala Likert 2
1. Siempre demostrado
2. Frecuentemente demostrado
3. A veces demostrado
4. Raramente demostrado
5. Nunca demostrado

\section{Escala Likert 1. a \\ 1. Ninguno \\ 2. Escaso \\ 3. Moderado \\ 4. Sustancial \\ 5. Extenso}

ANEXO 2. Modelo de registro del PCE

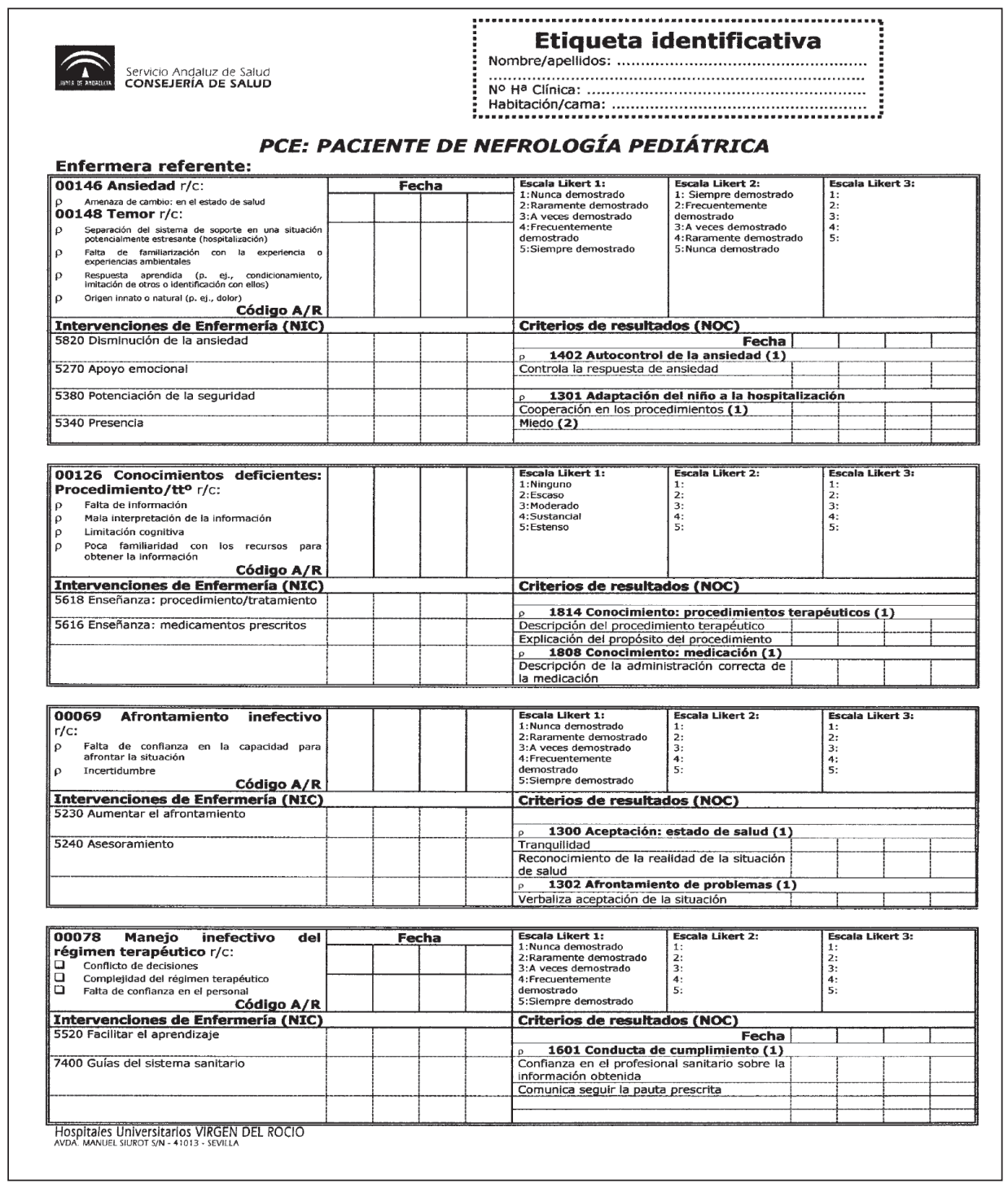


DIAGNÓSTICOS DEL PLAN DE CUIDADOS ESTANDARIZADO

\section{ANSIEDAD (00146)}

Vaga sensación de malestar o amenaza acompañada de una respuesta autonómica (cuyo origen con frecuencia es desconocido para el individuo); sentimiento de aprensión causado por la anticipación de un peligro. Es una señal de alerta que advierte de un peligro inminente y permite al individuo tomar medidas para afrontarlo.

Criterios de resultados: Autocontrol de la ansiedad (1402).

Indicadores: Controla la respuesta de ansiedad (140217). Escala de medida del indicador Likert 1

Intervenciones de enfermería: Disminución de la ansiedad (5820). Apoyo emocional (5270).

\section{TEMOR (00148)}

Respuesta a la percepción de una amenaza que se reconoce conscientemente como un peligro.

Criterios de resultados: Adaptación del niño a la hospitalización (1301).

Indicadores: Cooperación en los procedimientos (130118). Escala de medida del indicador: Likert 1. Miedo (130105). Escala de medida del indicador Likert 2

Intervenciones de enfermería: Potenciación de la seguridad (5380) y Presencia (5340).

\section{CONOCIMIENTOS DEFICIENTES (00126)}

Carencia o deficiencia de información cognitiva relacionada con un tema específico.

Criterios de resultados: Conocimiento: procedimientos terapéuticos (1814).

Indicadores: Descripción del procedimiento terapéutico (181401). Explicación del propósito del procedimiento (181402). Descripción de la administración correcta de la medicación (180810). Escala de medida del indicador: Likert 1 a.

Intervenciones de enfermería: Enseñanza: procedimiento/ tratamiento (5618). Enseñanza: Medicamentos prescritos (5616).

\section{AFRONTAMIENTO INEFECTIVO (00069)}

Incapacidad para llevar a cabo una apreciación válida de los agentes estresantes, para elegir adecuadamente las respuestas habituales o para usar los recursos disponibles.

Criterios de resultados: Aceptación: Estado de salud (1300). Afrontamiento de problemas (1302).
Indicadores: (130001) Tranquilidad. (130008) Reconocimiento de la realidad de la situación de salud. Verbaliza aceptación de la situación (130205).

Escala de medida del indicador: Likert 1

Intervenciones de enfermería: Aumentar el afrontamiento (5230). Asesoramiento (5240).

\section{MANEJO INEFECTIVO DEL RÉGIMEN TERA- PÉUTICO (00078)}

Incapacidad del paciente renal para cumplir el tratamiento, no alcanzando objetivos específicos de salud. Criterios de resultados: Conducta de cumplimiento (1601).

Indicadores: Confianza en el profesional sanitario sobre la información obtenida (160101). Solicita seguir la pauta prescrita (160102). Escala de medida del indicador: Likert 1

Intervenciones de enfermería: Facilitar el aprendizaje (5520). Guías del sistema sanitario (7400).

\section{RIESGO DE TRASTORNO DE LA IMAGEN COR- PORAL (00118)}

Confusión en la imagen mental del yo físico.

Criterios de resultados: Imagen corporal (1200). Indicadores: Adaptación a cambios en el aspecto físico (120007). Voluntad para utilizar estrategias que mejoren el aspecto y la función (120010). Escala de medida del indicador: Likert 1

Intervenciones de enfermería: Potenciación de la imagen corporal (5220).

\section{PROBLEMAS INTERDISCIPLINARES}

\section{COMPLICACIÓN POTENCIAL: FIEBRE.}

Intervención de enfermería: Tratamiento de la fiebre (3740).

\section{COMPLICACIÓN POTENCIAL: INFECCIÓN.}

Intervenciones de enfermería: Protección contra la infecciones (6550). Control de infecciones (6540). Vigilancia (6550). Mantenimiento de dispositivos de acceso venoso (2440). Cuidados del catéter urinario (1876).

\section{COMPLICACIÓN POTENCIAL: ESTREÑIMIEN- TO.}

Intervenciones de enfermería: Entrenamiento intestinal (0440). Manejo de la nutrición (1100). 


\section{COMPLICACIÓN POTENCIAL: DESEQUILI- BRIO HÍDRICO.}

Intervenciones de enfermería: Manejo de líquidos (4110).

\section{COMPLICACIÓN POTENCIAL: ALTERACIÓN NUTRICIONAL.}

Intervenciones de enfermería: Manejo de la nutrición (1100).

Un Plan de Cuidados Estandarizado define la aportación enfermera al cuidado de la salud del paciente. Fija indicadores que permiten valorar la calidad, estableciendo estándares de proceso y criterios de resultados. El diseño de este plan de cuidados nos ha permitido acordar, pactar y ajustar acciones a una situación que favorezca la disminución de la variabilidad en la práctica clínica. No debemos olvidar, no obstante, que el Plan de Cuidados Estandarizado del paciente de nefrología pediátrica sirve de base a partir del cual el enfermero valorará e identificará las necesidades individuales de cada paciente.

También debemos destacar que dadas las especiales características de nuestra Unidad, en donde se concentran todas las posibles terapéuticas a nivel de la patología renal (insuficiencia renal en prediálisis, diálisis peritoneal, hemodiálisis y trasplante renal), ya se encuentra definidos y en fase de acreditación planes de cuidados estandarizados específicos para cada una de estos procesos. Así como de la aplicación informática de dichos planes a corto plazo, lo que redundará en la sistematización de los mismos.

\section{Agradecimientos}

A todos aquellos que creen que la curiosidad, la inquietud, el trabajo y el afán de evolucionar y crecer en conocimientos para una mayor profesionalidad es una forma de ser.

\section{Bibliografía}

1. Alfaro-LeFevre R. Aplicación del proceso enfermero. Fomentar el cuidado en colaboración. $5^{a} \mathrm{Ed}$. Barcelona: Masson; 2005.

2. Charrier, J. Ritter, B. El Plan de Cuidados Estandarizado. Un soporte del diagnóstico enfermero. Elaboración y puesta en práctica. Ed. Masson. Elsevier España. 2005. Madrid.

3. Gordon, M. Manual de Diagnósticos de Enfermería. $11^{a}$ Ed. MacGraw-Hill. 2007.

4. Luis Rodrigo, M.T. Los diagnósticos enfermeros. Revisión crítica y guía práctica. $3^{\mathrm{a}} \mathrm{Ed}$. Barcelona: Masson; 2005.

5. McCloskey Dochterman, J. Bulechek, G. editors. Clasificación de intervenciones de enfermería (NIC). $4^{a}$ Ed. Madrid: Elsevier España; 2005.

6. Moorhead, S. Jonson, M. Maas, M. editors. Clasificación de resultados de enfermería (NOC). $3^{\mathrm{a}}$ Ed. Madrid: Elsevier España; 2005.

7. NANDA. Diagnósticos Enfermeros: Definiciones y clasificación 2005-2006. Madrid: Elsevier España; 2005. 